Chinese Journal of Organic Chemistry

ARTICLE

\title{
水相中可见光催化卤代芳烃羟基化反应
}

\author{
许秀枝 ${ }^{*}, a$ 张 帆 ${ }^{b}$ 黄 胜 $^{a}$ 张志强 ${ }^{a}$ 柯 方*,a \\ $\left({ }^{a}\right.$ 福建医科大学药学院 福建省天然药物药理学重点实验室 福州 350004) \\ ( ${ }^{b}$ 福建医科大学附属第一医院肿瘤内科 福州 350005)
}

\begin{abstract}
摘要 通过在可见光下卤代芳烃的差基化反应合成一系列苯酚类化合物. 反应用单质碘作光催化剂, 在室温下反应获 得较好收率的产物, 生成目标产物最高产率可达到 $92 \%$, 该体系对氯代芳烃同样具有较好的催化活性. 此方法具有便 宜、易处理、对环境无污染的优点，为酚类化合物的合成提供了一种简便经济的方法. 运用此策略，以 5-乙酰-4-溴-2甲氧基苄基二异丙基氨基二硫代甲酸酯合成的 5-乙酰-4-羟基-2-甲氧基苄基二异丙基氨基二硫代甲酸酯，具有明显的 抗增殖作用.
\end{abstract}

关键词 可见光; 单质碘; 卤代芳烃; 羊基化

\section{Visible-Light Promoted Hydroxylation of Aryl Halides under Mild Reaction Conditions in Neat Water}

\author{
$\mathrm{Xu}$, Xiuzhi $^{*, a} \quad{\text { Zhang, } \text { Fan }^{b} \quad \text { Huang, Sheng }^{a} \quad \text { Zhang, Zhiqiang }^{a} \quad \text { Ke, Fang }}^{\text {*,a }}$ \\ ( ${ }^{a}$ Fujian Provincial Key Laboratory of Natural Medicine Pharmacolog, Department of \\ Pharmacy, Fujian Medical University, Fuzhou 350004) \\ $\left({ }^{b}\right.$ The First Affiliated Hospital of Fujian Medical University Oncology, Fuzhou 35000)
}

\begin{abstract}
A novel visible-light-introduced reaction for the construction of phenols via hydroxylation of aryl halides has been developed. The reaction has been achieved in high yield under mild conditions by using iodine as photocatalyst, which is cheap, easy to handle and environmentally friendly. A variety of phenols were obtained in up to $92 \%$ yields. Moreover, aryl chlorides were also successfully employed as substrates, affording the target phenols in good isolated yields. It might provide promising protocol for the synthesis of phenol derivatives. Its application was performed by the synthesis of 5-acetyl-4-hydroxy-2-methoxybenzyl diisopropylcarbamodithioate, which displayed significant anti-proliferation effect.
\end{abstract}

Keywords visible-light promoted; iodine; aryl halides; hydroxylation

酚类化合物是有机化合物中一类十分重要的结构 单元，既是药物合成中的反应前体和中间体，又广泛存 在于天然产物分子和药物活性分子中 ${ }^{[1]}$. 如辉瑞公司生 产的 Pristiq, 通过影响对抑郁症起关键作用的两种物质 神经递质血清素和去甲肾上腺素的水平,从而到达抗抑 郁作用; Dnunorubicin 是人工合成的用来治疗急性淋巴 细胞性白血病的抑制剂(图 1). 因此, 制备酚类结构化合 物具有很高的合成价值 ${ }^{[2]}$.

早期大量的苯酚主要通过芳香烃的氯化/磺化等串 联反应得到 ${ }^{[3]}$, 在 1865 年之前, 虽然 Kekule 还未提出苯 的结构, 但用分子氧氧化苯环制备苯酚的研究已引起当<smiles>CN(C)CC(c1ccc(O)cc1)C1(O)CCCCC1</smiles>

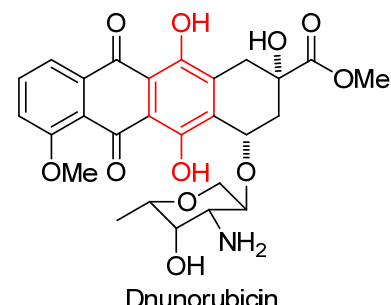

图 1 一些具有羟基结构的活性分子

Figure 1 Some active molecules with hydroxyl moieties

时人们的重视，目前全球超过 $90 \%$ 的苯酚是由异丙基苯 通过过氧化作用去烷基化制备 ${ }^{[4]}$; 此外 Taddei 等 ${ }^{[5]}$ 在 1986年报道了芳烃卤代物在双 (三甲基硅基)过氧化物的

* Corresponding authors. E-mail: xiuzhi_xu@126.com; kefang612@163.com

Received May 2, 2020; revised June 12, 2020; published online July 15, 2020.

Project supported by the Natural Science Foundation of Fujian Province (Nos. 2016Y9052, 2016Y9053, 2017J01820, 2017-1-64).

福建省自然科学基金(Nos. 2016Y9052, 2016Y9053, 2017J01820, 2017-1-64)资助项目. 
催化下制备苯酚的过程. 虽然这些方法很大程度上推动 了苯酚及其衍生物的工业合成发展, 但是这类方法存在 条件苛刻等缺点(Scheme 1a). 其次过渡金属催化可在相 对温和的反应条件下实现苯酚及其类似物的制备 ${ }^{[6]}$, 这 为卤代芳烃羟基化提供了新的途径(Scheme 1b). 2017 年, Fier 等 ${ }^{[7]}$ 报道了以 $\mathrm{CuX}$ 作为催化剂与氨基酸配体联 合作用下，催化卤代芳烃及亚胺类化合物合成酚类化合 物的方法，但是适用于该体系的配体难以制备. 此外 2018 年, Zhang 小组 ${ }^{[8]}$ 报道了介孔多氧金属酸盐离子杂 化剂[Pxy Dim $]_{2.5} \mathrm{PMoV}_{2}$ 作为多相催化剂来催化芳烃羟 基化生成苯酚的方法. 虽然过渡金属催化体系研究已有 较大的发展, 能高效合成苯酚及其衍生物, 但在一定程 度上依然存在着部分反应温度要求较高, 对温度敏感的 底物催化效果不佳等缺点, 不利于苯酚的简单、绿色合 成.

(a)<smiles>c1ccccc1</smiles>

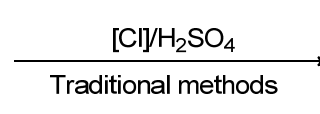

(b)
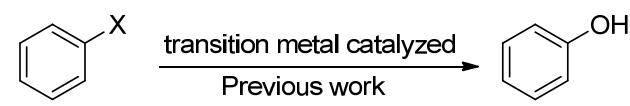

(c)

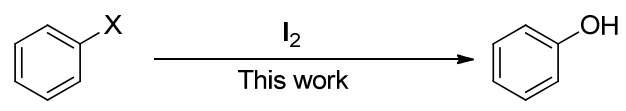

图式 1 酚类化合物的合成方法

Scheme 1 Strategies for the synthesis of phenols

最近, 光催化合成开始复兴, 由于其具有经济环保 性、反应条件温和等特点, 展示出了广阔的应用前景 ${ }^{[9]}$. 在有机光合成领域, 近几年发展的基于光催化 $\mathrm{C}-\mathrm{X}$ 官 能化的反应, 特别是通过 $\mathrm{C}-\mathrm{X}$ 构建 $\mathrm{C}-\mathrm{O}$ 键, 受到人们 的关注 ${ }^{[10]}$. 例如 $\mathrm{Fu}$ 等 ${ }^{[11]}$ 在 2016 年报道了可见光参与的 氧化差化反应, 该体系以卤代芳烃为起始原料, fac$\operatorname{Ir}(\mathrm{ppy})_{3}$ 作为光催化剂, 得到了相应的酚类化合物. 其次 水作为环境友好的载体, 与对人体有害的有机溶剂相 比, 是反应中最安全无毒的溶剂. 价廉的水可以让化学 反应过程更符合经济性和 “绿色化学” 的原则. 经过近 几年的发展, 水相有机化学反应引起了广大化学家的关 注 $^{[12]}$. 因此研究和发展高效、简单、水相中的卤代芳烃 羟基化反应, 改善传统合成条件的缺陷和不足, 实现该 类反应的绿色合成, 拓宽其底物适应性, 对有机合成的 发展具有重要的意义.

尽管这一领域已取得一些进展, 但是目前可见光催 化卤代烃的羟基化反应的相关报道仍旧很少 ${ }^{[13]}$. 最近 Cai 课题组 ${ }^{[10 \mathrm{a}]}$ 在 2019 年报道的有机卤化物的光致着基 化反应, 为合成一系列非官能化酚类和脂肪醇类化合物 提供了新的启发, 但是微毒的还原剂三乙胺和反应体系 中所用到的有机溶剂乙腈限制了其的发展. 基于本课题
组前期对环境友好型催化体系的研究 ${ }^{[14]}$, 我们提出用 一种廉价易得的单质碘来作为光敏催化剂, 卤代芳烃为 原料, 只用 $25 \mathrm{~W}$ 的白色节能灯在水相中高效地进行碘 代芳烃的羟基化反应，合成一系列苯酚衍生物，而且对 较不活泼的溴代芳烃，特别是氯代芳烃同样适用，具有 潜在的应用价值, 为酚类化合物的合成提供了一种简便 经济的方法(Scheme 1c).

\section{1 结果与讨论}

\section{1 反应条件的优化}

以碘苯作为反应底物, 通过对光敏剂、助剂、碱和 反应溶剂等条件的篮选来确定最优反应条件，结果如表 1 所示. 当使用有机光敏剂曙红(Eosin Y)时, 在 $25 \mathrm{~W}$ 的 白色节能灯照射下，反应只能获得 $22 \%$ 的收率. 然而当 使用单质碘 $\left(\mathrm{I}_{2}\right)$ 时，则展现了最佳催化效果，获得 $60 \%$ 的 收率. 使用有机光催化剂甲基橙(Methyl Orange)时, 却 得不到预期效果(表 1, Entries 1 3). 同时，做了相关的 控制实验，当反应中缺少光照或缺少曙红 Y 时，检测不 到反应产物(表 1, Entry 4). 随后, 以 $\mathrm{I}_{2}$ 作为光催化剂, 考察了不同助剂的催化效果，如四丁基溴化铵(TBAB)、 四丁基碘化铵(TBAI)和 KI 等，结果表明 TBAI 表现出最 好的催化效果(表 1, Entries 5 8). 对碱进行笁选, 发现 $\mathrm{NaOH}$ 作碱时，反应能以高达 $91 \%$ 的分离收率得到目标 化合物, 而其他强碱或弱碱并不能得到较好的收率(表 1, Entries 9 12). 溶剂对反应产率有着极大的影响: 在 四氢呋喃(THF)、乙腈 $\left(\mathrm{CH}_{3} \mathrm{CN}\right) 、$ 二甲亚砜(DMSO)、 $N, N-$ 二甲基甲酰胺(DMF)中仅能观测到痕量的产物生成(表 1, Entries 14 17), 而在甲醇(表 1, Entry 13)和纯水中, 分别以 $87 \%$ 和 $91 \%$ 的产率得到产物. 最后以纯水为最佳 溶剂. 因此, 从实验结果可以得出, 该反应体系的最佳 催化条件为: 碘苯 $(0.5 \mathrm{mmol}), \mathrm{I}_{2}(5 \mathrm{~mol} \%) 、 \mathrm{NaOH}(2$ $\mathrm{mmol}) 、 \mathrm{TBAI}(0.05 \mathrm{mmol})$, 加入 $2 \mathrm{~mL}$ 水作为溶剂, $25 \mathrm{~W}$ 可见光共同作用下反应 $24 \mathrm{~h}$.

\section{2 反应底物范围的拓展}

为了探索该催化体系的适用范围, 在最佳反应条件 下探究了不同取代基对反应的适用性，结果如表 2 所示. 实验表明：碘代芳烃的活性大于溴代芳烃，且大于氯代 芳烃. 如碘苯制备苯酚，能得到 $91 \%$ 的收率; 溴苯只能 得到 $83 \%$ 的收率; 而氯苯只能得到 $72 \%$ 的收率(表 $2, \mathbf{2 a}$ ). 考察了具有不同取代基电子效应的底物 1 在该反应条件 下的适用性(表 2). 当底物 $\mathbf{1}$ 的苯环上不含取代基时, 目 标产物的收率为 91\%(表 2, 2a). 当底物 1 的苯环对位带 有各种给电子基团，如甲基、甲氧基或羟基时，反应都 能顺利进行，并以良好产率获得相应的目标羟基化产物 (表 $2, \mathbf{2} \mathbf{b} \sim 2 \mathbf{d}$ ). 而且, 改变取代基的位置对反应收率没 
表 1 反应条件的优化 ${ }^{a}$

Table 1 Optimization of the reaction conditions

\begin{tabular}{|c|c|c|c|c|c|}
\hline Entry & Cat. & PTC & Base & Solvent & Yield $^{b} / \%$ \\
\hline 1 & $\operatorname{Eosin} Y$ & $\mathrm{NaI}$ & $\mathrm{NaOH}$ & $\mathrm{H}_{2} \mathrm{O}$ & 22 \\
\hline 2 & Methyl orange & $\mathrm{NaI}$ & $\mathrm{NaOH}$ & $\mathrm{H}_{2} \mathrm{O}$ & 0 \\
\hline 3 & $\mathrm{I}_{2}$ & $\mathrm{NaI}$ & $\mathrm{NaOH}$ & $\mathrm{H}_{2} \mathrm{O}$ & 60 \\
\hline 4 & - & $\mathrm{NaI}$ & $\mathrm{NaOH}$ & $\mathrm{H}_{2} \mathrm{O}$ & Trace \\
\hline 5 & $\mathrm{I}_{2}$ & - & $\mathrm{NaOH}$ & $\mathrm{H}_{2} \mathrm{O}$ & 28 \\
\hline 6 & $\mathrm{I}_{2}$ & KI & $\mathrm{NaOH}$ & $\mathrm{H}_{2} \mathrm{O}$ & 46 \\
\hline 7 & $\mathbf{I}_{2}$ & TBAI & $\mathrm{NaOH}$ & $\mathrm{H}_{2} \mathrm{O}$ & 91 \\
\hline 8 & $\mathrm{I}_{2}$ & TBAB & $\mathrm{NaOH}$ & $\mathrm{H}_{2} \mathrm{O}$ & 82 \\
\hline 9 & $\mathrm{I}_{2}$ & TBAI & - & $\mathrm{H}_{2} \mathrm{O}$ & 0 \\
\hline 10 & $\mathrm{I}_{2}$ & TBAI & $\mathrm{KOH}$ & $\mathrm{H}_{2} \mathrm{O}$ & 84 \\
\hline 11 & $\mathrm{I}_{2}$ & TBAI & $\mathrm{Cs}_{2} \mathrm{CO}_{3}$ & $\mathrm{H}_{2} \mathrm{O}$ & 81 \\
\hline 12 & $\mathrm{I}_{2}$ & TBAI & $\mathrm{K}_{2} \mathrm{CO}_{3}$ & $\mathrm{H}_{2} \mathrm{O}$ & 71 \\
\hline 13 & $\mathrm{I}_{2}$ & TBAI & $\mathrm{NaOH}$ & $\mathrm{CH}_{3} \mathrm{OH}$ & 87 \\
\hline 14 & $\mathrm{I}_{2}$ & TBAI & $\mathrm{NaOH}$ & THF & 32 \\
\hline 15 & $\mathrm{I}_{2}$ & TBAI & $\mathrm{NaOH}$ & $\mathrm{CH}_{3} \mathrm{CN}$ & 25 \\
\hline 16 & $\mathrm{I}_{2}$ & TBAI & $\mathrm{NaOH}$ & DMSO & 36 \\
\hline 17 & $\mathrm{I}_{2}$ & TBAI & $\mathrm{NaOH}$ & DMF & 42 \\
\hline
\end{tabular}

${ }^{a}$ Reaction conditions: 1,2 -iodobenzene $(0.5 \mathrm{mmol})$, photo catalyst $(5 \mathrm{~mol} \%)$, base ( $1 \mathrm{mmol})$, PTC ( $0.05 \mathrm{mmol})$, and $\mathrm{H}_{2} \mathrm{O}(2 \mathrm{~mL}), 25 \mathrm{~W}$ LED, $24 \mathrm{~h} ;{ }^{b}$ Isolated yield.

有明显影响(表 $2, \mathbf{2 b} \sim 2 \mathbf{r}$ ). 但是, 当苯环上含有多个强 给电子基团时, 目标产物收率有所下降, 这可能是由于 底物卤代烃被过度氧化所致(表 $2,2 \mathbf{v}, 2 \mathbf{w}$ ). 另外，当底 物 1 的苯环上含有不同卤素取代基(如氯、溴或氟)时，均 以中等收率得到目标羟基化产物(表 $2, \mathbf{2} \mathbf{j} \sim \mathbf{2 l}$ ). 此外, 还试验了其它含有强吸电子取代基(如硝基、羧基、乙 酰基和氧基等)的底物, 羟基化反应活性较差(表 $2,2 \mathrm{e} \sim$ 2i). 这些实验结果说明取代基的电子效应对该反应具 有一定影响. 考察了杂环芳烃底物的羟基化反应的适用 性(表 2, 2s), 也能较好的完成此反应进程, 得到相对较 好的收率.

\section{3 克级反应}

依据最优反应条件对底物进行克级放大实验. 以碘 苯 (5 mmol) 为原料, $\mathrm{I}_{2}(5 \mathrm{~mol} \%)$ 为催化剂, $20 \mathrm{mmol}$ $\mathrm{NaOH}$ 为碱, $0.5 \mathrm{mmol} \mathrm{TBAI}$ 为添加剂, $20 \mathrm{~mL}$ 水为溶剂, $25 \mathrm{~W}$ LED 白光照射下反应 $30 \mathrm{~h}$, 顺利合成苯酚, 产率 为 $73 \%$.

\section{4 活性实验}

丹皮酚称牡丹酚或苟药酮. 药理实验证明, 其具有 抗心律失常、抗动脉弹样硬化、改善微循环、保护缺血 组织等作用. 同时药理研究还发现丹皮酚及其衍生物也 对食道癌、肝癌、结肠癌、白血病等均有明显的抑制作 用 ${ }^{[15]}$. 本方法为合成丹皮酚及其衍生物提供了一种新 的合成策略. 例如 5-乙酰-4-羟基-2-甲氧基苄基二
表 2 苯酚衍生物的合成 ${ }^{a, b}$

Table 2 Synthesis of phenols
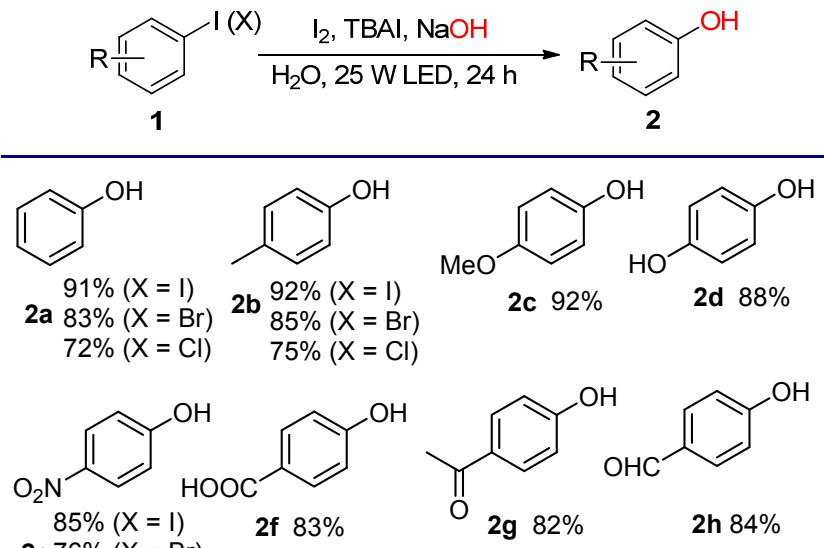

2e $76 \%(X=B r)$

$69 \%(X=\mathrm{Cl})$
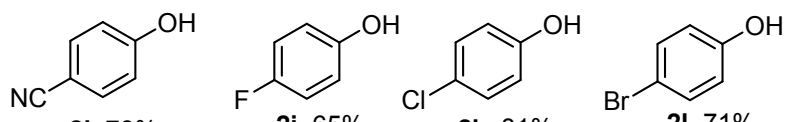<smiles>Oc1ccccc1O</smiles><smiles>O=[N+]([O-])c1ccccc1O</smiles>

2m 93\% 2n $78 \%$<smiles>Nc1ccccc1O</smiles><smiles>Cc1ccccc1O</smiles>

2p $90 \%$<smiles>COc1ccccc1O</smiles><smiles>O=C(O)c1ccccc1O</smiles>
2r $75 \%$<smiles>Oc1cccnc1</smiles>
2s $81 \%$<smiles>COc1cccc(O)c1</smiles><smiles>Cc1cc(O)cc(O)c1</smiles><smiles>Cc1ccc(O)cc1C</smiles>

2w $84 \%$ 2v $83 \%$<smiles>[14CH3]c1cccc(O)c1</smiles>

${ }^{a}$ Reaction conditions: aryl halide $(0.5 \mathrm{mmol}), \mathrm{I}_{2}(5 \mathrm{~mol} \%), \mathrm{NaOH}(1 \mathrm{mmol})$, TBAI $(0.05 \mathrm{mmol})$, and $\mathrm{H}_{2} \mathrm{O}(2 \mathrm{~mL}), 25 \mathrm{~W}$ LED, $24 \mathrm{~h} ;{ }^{b}$ Isolated yield

异丙基氨基二硫代甲酸酯以 5-乙酰-4-溴-2-甲氧基芐基 二异丙基氨基二硫代甲酸酯为起始原料的总收率为 73\% (Scheme 2). 然后将所得化合物 A2 用于噻唑蓝 (MTT)分析，结果如图 2 所示. 其具有对人非小细胞肺 癌细胞 NCI-H1299 和宫颈癌细胞 Hela 的抗增殖作用. 针对 NCI-H1299 细胞和 Hela 细胞的 $\mathrm{IC}_{50}$ 值检测结果分 别为 12.29 和 $14.46 \mu \mathrm{g} / \mathrm{mL}$ (图 2) ${ }^{[16]}$.

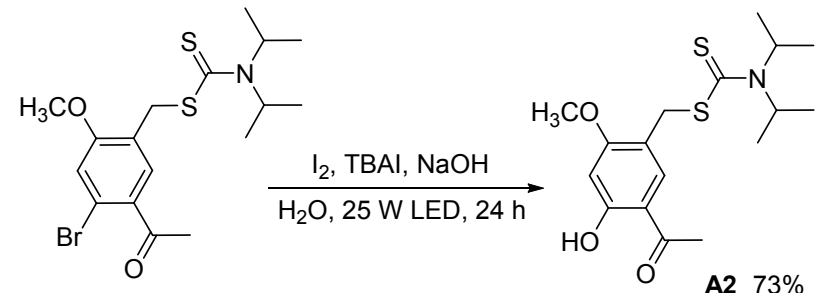

图式 2 5-乙酰-4-羟基-2-甲氧基苄基二异丙基氨基二硫代甲 酸酯的合成

Scheme 2 Synthesis of 5-acetyl-4-hydroxy-2-methoxybenzyl diisopropylcarbamodithioate in water 

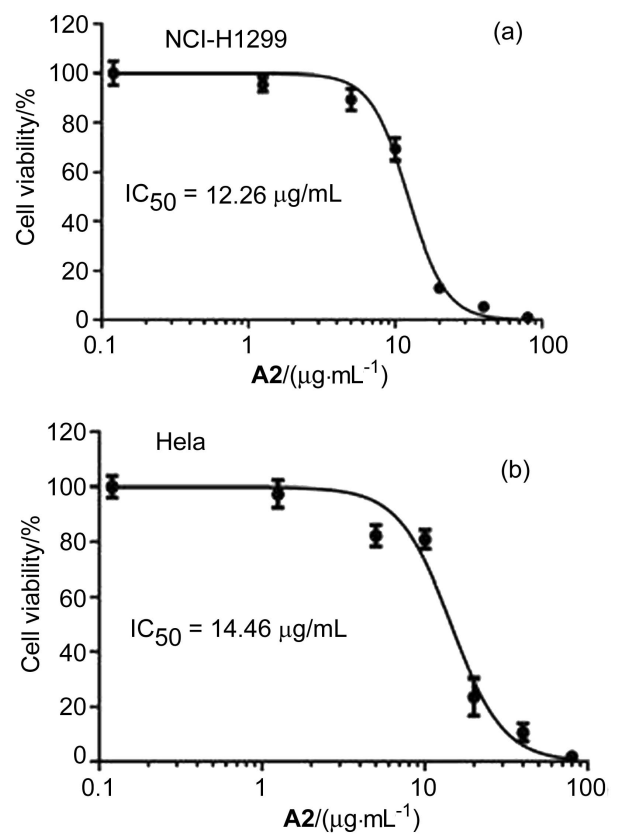

图 2 NCI-H1299 和 Hela 细胞抑制率与初始浓度的关系

Figure 2 Relationship between inhibition rate for NCI-H1299 and Hela cells and initial concentration

\section{5 机理探索}

为了进一步探讨反应机理, 在水中进行了循环伏安 $(\mathrm{CV})$ 实验, 以 $\mathrm{TBAI}$ 为电解质, 以玻璃电极为工作电极, 铂丝为对电极, $\mathrm{SCE}$ 为参比电极, 扫描速率为 $0.1 \mathrm{~V} \cdot \mathrm{s}^{-1}$ (图 3). 通过比较曲线 $\mathrm{A}$ 和 $\mathrm{B}$, 当加入硔单质时 $i_{\mathrm{pa}}$
从 $2.3 \mathrm{~mA}$ 增加到 $4.8 \mathrm{~mA}$, 并且曲线 $\mathrm{D}$ 表明碘苯和碘呈 可逆氧化还原过程. 实验结果表明碘单质起催化作用且 在反应过程中再生.

\section{6 反应机理}

根据文献报道 ${ }^{[10 a, 13,17]}$ 和以上实验结果, 提出了一个 可能的实验机理, 如图 3 所示. 碘单质在碱性条件下容 易发生歧化反应生成具有强氧化性质的 $\mathrm{NaIO}_{3}$ 和常见的 $\mathrm{NaI}$, 通常情况下这个反应过程较为缓慢, 我们利用光 照来加速这个过程. 首先原料碘苯在碱性条件下使碘离 子游离产生中间体 $\mathbf{A}$, 然后中间体 $\mathbf{A}$ 在 $\mathrm{NaIO}_{3}$ 的氧化作 用下产生具有氧化性质的中间体 B. 通常碘化钠在空气 中容易被氧气缓慢地氧化生成碘单质，如果在碱性条件 下反应速度会适当提高. 在此实验中碘化钠在接触具有 氧化性质的中间体 $\mathbf{B}$ 时被氧化生成单质碘，中间体 $\mathbf{B}$ 接 受质子生成最终产物(Scheme 3).

\section{2 结论}

通过用单质硒作光催化剂实现了以卤代芳烃为原 料, 以 $\mathrm{NaOH}$ 为氧源，水相中羟基化反应合成苯酚衍生 物的方法. 反应用便宜、易处理、对环境无污染的单质 碘作为光催化剂, 利用纯水作为溶剂, 较大地降低了反 应成本. 该体系对较不活泼的溴代和氯代芳烃同样适 用，具有较大的潜在应用价值，为一系列通过卤代芳烃 合成苯酚衍生物的合成提供了一种更简便经济的方法,

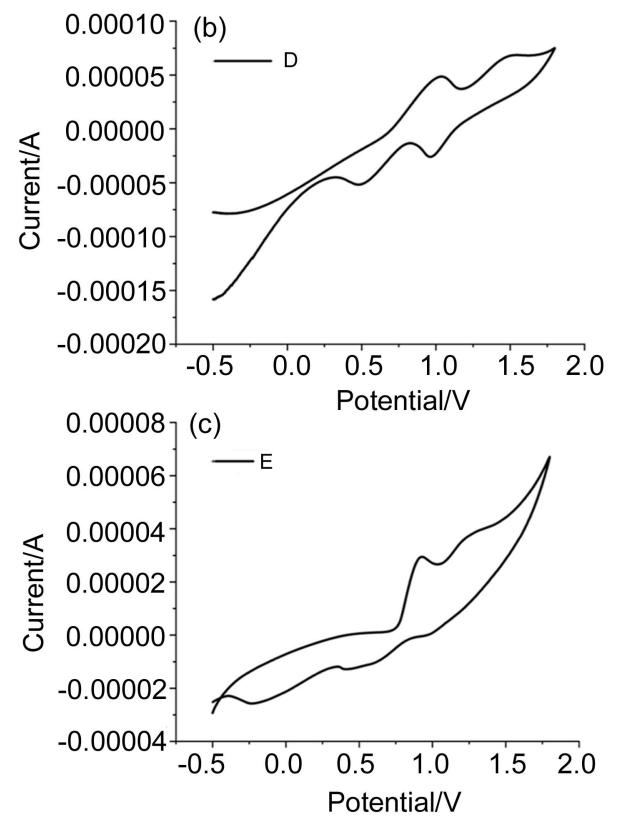

图 3 循环伏安实验

Figure 3 Cyclic voltammogram

A: Iodobenzene $+\mathrm{NaOH}+\mathrm{TBAI}$; : Iodobenzene $+\mathrm{NaOH}+\mathrm{TBAI}+\mathrm{I}_{2} ; \mathrm{C}$ : Iodobenzene $+\mathrm{NaOH}+\mathrm{I}_{2} ; \mathrm{D}$ : Iodobenzene $+\mathrm{I}_{2} ; \mathrm{E}$ : Iodobenzene $+\mathrm{I}_{2}+\mathrm{TBAI}$ 


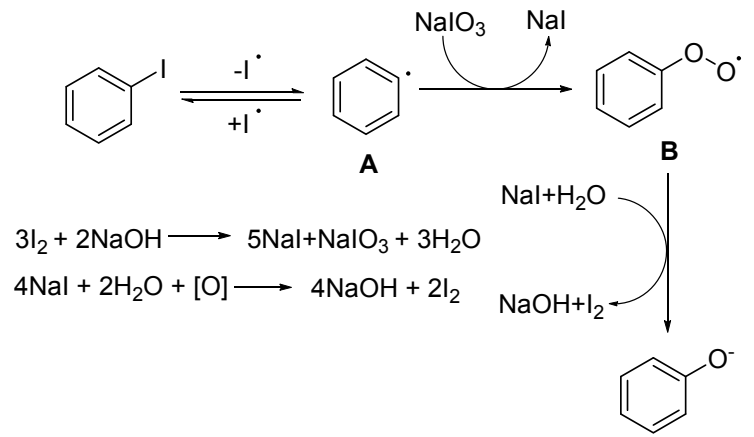

图式 3 自由基捕获实验

Scheme 3 Radical trapping experiments

更多的关于可见光催化的羟基化反应的研究在我们实 验室进行中.

\section{3 实验部分}

\section{1 仪器与试剂}

光催化反应采用 $25 \mathrm{~W}$ 白色 LED 进行光照射. 反应 体系采用北京 Perfectlight PCX50C Discover 进行. Agilent $6890 \mathrm{~N}$ 质谱仪进行气相色谱/质谱(GC/MS) 测试 [5973N 质量检测器(EI)和 HP5-MS $30 \mathrm{~m} \times 0.25 \mathrm{~mm}$ 毛细 管非极性柱]; Bruker AM 400 核磁共振仪, 以 TMS 为内 标, 氛代氯仿、DMSO 等気代试剂为溶剂; X-4 型数字熔 点仪. 柱色谱用硅胶(300 400 目)进行, 乙酸乙酯和石 油醚为洗脱剂. 硅胶 GF254 板进行薄层色谱. 所用的 药品和试剂均为市售分析纯或化学纯, 除特别说明外, 未经进一步处理.

\section{2 实验方法}

在 $10 \mathrm{~mL}$ 的圆底烧瓶中加入碘苯 $(0.5 \mathrm{mmol}), \mathrm{I}_{2}$ (0.05 equiv.), $\mathrm{NaOH}(2 \mathrm{mmol}), \mathrm{TBAI}(0.05 \mathrm{mmol})$ 和溶剂 $\mathrm{H}_{2} \mathrm{O}(2 \mathrm{~mL}$ ). 反应液在一个 $25 \mathrm{~W}$ 的白光 LED 灯下(距离 反应瓶 $5 \mathrm{~cm}$ ) 室温搅拌 $24 \mathrm{~h}$. 反应用薄层色谱(TLC)检 测. 反应完全后酸化, 调节至 $\mathrm{pH}=6$, 减压蒸馏, 粗产 品通过柱层析分离纯化 $[V$ (乙酸乙酯) $: ~ V$ (石油醚 $)=1$ : 4]得到目标产物 (温水浴条件下减压蒸馏), 计算产率. 目标产物通过 ${ }^{1} \mathrm{H} N M R,{ }^{13} \mathrm{C}$ NMR 和质谱进行结构表征. $\mathbf{2 a} \sim 2 \mathbf{w}$ 为已知化合物.

苯酚 $(2 a){ }^{[19]}$ : 白色固体, m.p. $42 \sim 44{ }^{\circ} \mathrm{C}$ (Lit. ${ }^{[1 f]}$ : $\left.43{ }^{\circ} \mathrm{C}\right) ;{ }^{1} \mathrm{H}$ NMR $\left(400 \mathrm{MHz}, \mathrm{CDCl}_{3}\right) \delta: 7.23 \sim 7.20(\mathrm{~m}$, $2 \mathrm{H}), 6.94 \sim 6.90(\mathrm{~m}, 1 \mathrm{H}), 6.84 \sim 6.81(\mathrm{~m}, 2 \mathrm{H}), 5.64(\mathrm{~s}$, $1 \mathrm{H}) ;{ }^{13} \mathrm{C}$ NMR $\left(100 \mathrm{MHz}, \mathrm{CDCl}_{3}\right) \delta: 155.4,129.8,121.0$, 115.5; MS (EI) $m / z: 94\left[\mathrm{M}^{+}\right]$.

对甲基苯酚 (2b) ${ }^{[19]}$ : 白色固体, m.p. $33 \sim 34{ }^{\circ} \mathrm{C}$ (Lit. $\left.{ }^{[1 \mathrm{ff}]}: 35{ }^{\circ} \mathrm{C}\right)$; ${ }^{1} \mathrm{H}$ NMR (400 MHz, $\left.\mathrm{CDCl}_{3}\right) \delta: 7.08 \sim$ $7.05(\mathrm{~m}, 2 \mathrm{H}), 6.80 \sim 6.76(\mathrm{~m}, 2 \mathrm{H}), 5.64(\mathrm{br}, 1 \mathrm{H}), 2.31(\mathrm{t}$,
$J=4.0 \mathrm{~Hz}, 3 \mathrm{H}) ;{ }^{13} \mathrm{C}$ NMR $\left(100 \mathrm{MHz}, \mathrm{CDCl}_{3}\right) \delta: 153.1$, 130.2, 115.7, 115.3, 20.5; MS (EI) $m / z: 108\left[\mathrm{M}^{+}\right]$.

对甲氧基苯酚 (2c) ${ }^{[19]}$ : 白色固体, m.p. $54 \sim 55{ }^{\circ} \mathrm{C}$ (Lit. $\left.{ }^{[1 \mathrm{f}]}: 56{ }^{\circ} \mathrm{C}\right) ;{ }^{1} \mathrm{H}$ NMR $\left(400 \mathrm{MHz}, \mathrm{CDCl}_{3}\right) \delta: 6.79 \sim$ $6.78(\mathrm{~m}, 4 \mathrm{H}), 5.45(\mathrm{br}, 1 \mathrm{H}), 3.77(\mathrm{~s}, 3 \mathrm{H}) ;{ }^{13} \mathrm{C}$ NMR $(100$ $\left.\mathrm{MHz}, \mathrm{CDCl}_{3}\right) \delta: 153.7,149.7,116.3,115.1,56.0 ; \mathrm{MS}(\mathrm{EI})$ $m / z: 124\left[\mathrm{M}^{+}\right]$.

对苯二酚 (2d) ${ }^{[18 a]}$ : 白色固体, m.p. $171 \sim 172{ }^{\circ} \mathrm{C}$ (Lit. $\left.{ }^{[18 \mathrm{a}]}: 171{ }^{\circ} \mathrm{C}\right) ;{ }^{1} \mathrm{H}$ NMR $\left(400 \mathrm{MHz}\right.$, DMSO- $\left.d_{6}\right) \delta: 8.61$ (s, 2H), 6.57 (s, 4H); ${ }^{13} \mathrm{C}$ NMR (100 MHz, DMSO- $\left.d_{6}\right) \delta$ : 149.8, 115.8; MS (EI) $m / z: 110\left[\mathrm{M}^{+}\right]$.

对硝基苯酚 $(2 \mathrm{e})^{[19]}$ : 黄色固体, m.p. $111 \sim 112{ }^{\circ} \mathrm{C}$ (Lit. ${ }^{[1 f]}: 112{ }^{\circ} \mathrm{C}$ ); ${ }^{1} \mathrm{H}$ NMR $\left(400 \mathrm{MHz}, \mathrm{CDCl}_{3}\right.$ ) $\delta: 8.17$ (d, $J=9.2 \mathrm{~Hz}, 2 \mathrm{H}), 6.93(\mathrm{~d}, J=9.2 \mathrm{~Hz}, 2 \mathrm{H}), 6.46(\mathrm{br}, 1 \mathrm{H}) ;{ }^{13} \mathrm{C}$ NMR $\left(100 \mathrm{MHz}, \mathrm{CDCl}_{3}\right) \delta: 161.8,141.9,126.5,115.9$; MS (EI) $m / z: 139\left[\mathrm{M}^{+}\right]$.

对羟基苯甲酸 $(\mathbf{2 f})^{[19]}$ : 白色固体, m.p. $213 \sim 215{ }^{\circ} \mathrm{C}$ (Lit. ${ }^{[12 \mathrm{~d}]}: 214 \sim 216{ }^{\circ} \mathrm{C}$ ); ${ }^{1} \mathrm{H}$ NMR (400 MHz, DMSO- $d_{6}$ ) $\delta: 12.40(\mathrm{br}, 1 \mathrm{H}), 10.20$ (br, 1H), $7.80(\mathrm{~d}, J=8.8 \mathrm{~Hz}, 2 \mathrm{H})$, $6.82(\mathrm{~d}, J=8.8 \mathrm{~Hz}, 2 \mathrm{H}) ;{ }^{13} \mathrm{C}$ NMR $\left(100 \mathrm{MHz}\right.$, DMSO- $\left.d_{6}\right)$ $\delta: 167.3,161.7,131.6,121.5,115.2$; MS (EI) $\mathrm{m} / z: 138$ $\left[\mathrm{M}^{+}\right]$.

对乙酰基苯酚 $(2 \mathrm{~g})^{[19]}$ : 白色固体, m.p. 109 $110{ }^{\circ} \mathrm{C}$ (Lit. ${ }^{[18 b]}: 110{ }^{\circ} \mathrm{C}$ ); ${ }^{1} \mathrm{H}$ NMR (400 MHz, $\mathrm{CDCl}_{3}$ ) $\delta: 8.24$ (br, 1H), 7.91 (d, $J=8.8 \mathrm{~Hz}, 2 \mathrm{H}), 6.96$ (d, $J=8.8 \mathrm{~Hz}, 2 \mathrm{H}), 2.59$ (s, 3H); ${ }^{13} \mathrm{C}$ NMR $\left(100 \mathrm{MHz}, \mathrm{CDCl}_{3}\right) \delta: 196.2,161.7$, 131.4, 129.6, 115.7, 26.4; MS (EI) $m / z: 136\left[\mathrm{M}^{+}\right]$.

对羟基苯甲醛(2h) ${ }^{[19]}$ : 白色固体, m.p. $112 \sim 113{ }^{\circ} \mathrm{C}$ (Lit. $\left.{ }^{[1 \mathrm{ff}]}: 113{ }^{\circ} \mathrm{C}\right) ;{ }^{1} \mathrm{H}$ NMR (400 MHz, DMSO- $\left.d_{6}\right) \delta: 10.58$ (s, 1H), $9.78(\mathrm{~s}, 1 \mathrm{H}), 7.75(\mathrm{~d}, J=8.7 \mathrm{~Hz}, 2 \mathrm{H}), 6.93(\mathrm{~d}, J=$ $8.5 \mathrm{~Hz}, 2 \mathrm{H}) ;{ }^{13} \mathrm{C}$ NMR (100 MHz, DMSO- $\left.d_{6}\right) \delta: 191.0$, 163.4, 132.1, 128.5, 115.9; MS (EI) $\mathrm{m} / z: 122\left[\mathrm{M}^{+}\right]$.

对氰基苯酚 (2i) ${ }^{[19]}$ : 白色固体, m.p. $110 \sim 111{ }^{\circ} \mathrm{C}$ (Lit. $\left.{ }^{[18 c]}: 110 \sim 112{ }^{\circ} \mathrm{C}\right) ;{ }^{1} \mathrm{H}$ NMR $\left(400 \mathrm{MHz}, \mathrm{CDCl}_{3}\right) \delta$ : $7.55(\mathrm{~d}, J=8.8 \mathrm{~Hz}, 2 \mathrm{H}), 6.95(\mathrm{~d}, J=8.8 \mathrm{~Hz}, 2 \mathrm{H}) ;{ }^{13} \mathrm{C}$ NMR $\left(100 \mathrm{MHz}, \mathrm{CDCl}_{3}\right) \delta: 160.5,134.5,119.4,116.6$, 102.9; MS (EI) $m / z: 119\left[\mathrm{M}^{+}\right]$.

对氟苯酚 $(2 \mathbf{j}){ }^{[19]}$ : 白色固体, m.p. $44 \sim 46{ }^{\circ} \mathrm{C}$ (Lit. $\left.{ }^{[1]]}: 45{ }^{\circ} \mathrm{C}\right) ;{ }^{1} \mathrm{H}$ NMR $\left(400 \mathrm{MHz}, \mathrm{CDCl}_{3}\right) \delta: 6.96 \sim$ $6.90(\mathrm{~m}, 2 \mathrm{H}), 6.82 \sim 6.77(\mathrm{~m}, 2 \mathrm{H}), 6.17(\mathrm{br}, 1 \mathrm{H}),{ }^{13} \mathrm{C} \mathrm{NMR}$ $\left(100 \mathrm{MHz}, \mathrm{CDCl}_{3}\right) \delta: 158.7,156.3,151.1,116.5,116.4$, 116.3, 116.1; MS (EI) $m / z: 112\left[\mathrm{M}^{+}\right]$.

对氯苯酚 $(\mathbf{2 k})^{[19]}$ : 白色固体, m.p. $43 \sim 45{ }^{\circ} \mathrm{C}$ (Lit. ${ }^{[12 \mathrm{~d}]}: 42 \sim 44{ }^{\circ} \mathrm{C}$ ); ${ }^{1} \mathrm{H}$ NMR $\left(400 \mathrm{MHz}, \mathrm{CDCl}_{3}\right) \delta: 7.19$ (d, $J=8.9 \mathrm{~Hz}, 2 \mathrm{H}), 6.77$ (d, $J=8.9 \mathrm{~Hz}, 2 \mathrm{H}), 5.36$ (br, 1H); 
${ }^{13} \mathrm{C}$ NMR (100 MHz, $\left.\mathrm{CDCl}_{3}\right) \delta: 154.1,129.7,125.9,116.8$; MS (EI) $m / z: 128\left[\mathrm{M}^{+}\right]$.

对溴苯酚 (2I) ${ }^{[19]}$ : 白色固体, m.p. $61 \sim 63{ }^{\circ} \mathrm{C}$ (Lit. $\left.{ }^{[12 \mathrm{~d}]}: 62 \sim 63{ }^{\circ} \mathrm{C}\right) ;{ }^{1} \mathrm{H}$ NMR $\left(400 \mathrm{MHz}, \mathrm{CDCl}_{3}\right) \delta: 7.33$ (d, $J=8.9 \mathrm{~Hz}, 2 \mathrm{H}), 6.72$ (d, $J=8.9 \mathrm{~Hz}, 2 \mathrm{H}), 5.09$ (br, $1 \mathrm{H}$ ); ${ }^{13} \mathrm{C}$ NMR $\left(100 \mathrm{MHz}, \mathrm{CDCl}_{3}\right) \delta: 154.6,132.6,117.3,113.1$; MS (EI) $m / z: 173\left[\mathrm{M}^{+}\right]$.

邻苯二酚 $(\mathbf{2 m})^{[19]}$ : 白色固体]; m.p. 104 106 ${ }^{\circ} \mathrm{C}$ (Lit. ${ }^{[18 \mathrm{~d}]}$ : $\left.105 \sim 108{ }^{\circ} \mathrm{C}\right) ;{ }^{1} \mathrm{H}$ NMR $\left(400 \mathrm{MHz}, \mathrm{CDCl}_{3}\right) \delta$ : $6.88 \sim 6.86(\mathrm{~m}, 2 \mathrm{H}), 6.84 \sim 6.80(\mathrm{~m}, 2 \mathrm{H}), 5.32(\mathrm{~s}, 2 \mathrm{H}) ;{ }^{13} \mathrm{C}$ NMR (100 MHz, $\left.\mathrm{CDCl}_{3}\right) \delta: 143.7,121.5,115.7 ; \mathrm{MS}(\mathrm{EI})$ $m / z: 110\left[\mathrm{M}^{+}\right]$.

邻硝基苯酚 (2n) ${ }^{[19]}$ : 黄色固体, m.p. $44 \sim 45{ }^{\circ} \mathrm{C}$ (Lit. $\left.{ }^{[18 \mathrm{e}]}: 43 \sim 44{ }^{\circ} \mathrm{C}\right) ;{ }^{1} \mathrm{H}$ NMR $\left(400 \mathrm{MHz}, \mathrm{CDCl}_{3}\right) \delta$ : $10.58(\mathrm{~d}, J=1.5 \mathrm{~Hz}, 1 \mathrm{H}), 8.11(\mathrm{~d}, J=8.5 \mathrm{~Hz}, 1 \mathrm{H}), 7.60 \sim$ $7.56(\mathrm{~m}, 1 \mathrm{H}), 7.16(\mathrm{~d}, J=8.5 \mathrm{~Hz}, 1 \mathrm{H}), 7.01 \sim 6.97(\mathrm{~m}$, $1 \mathrm{H}) ;{ }^{13} \mathrm{C}$ NMR $\left(100 \mathrm{MHz}, \mathrm{CDCl}_{3}\right) \delta: 155.3,137.7$, 130.5,125.2, 120.4, 120.1; MS (EI) $m / z: 139\left[\mathrm{M}^{+}\right]$.

邻氨基苯酚 (2o $)^{[19]}$ : 灰色固体, m.p. $171 \sim 173{ }^{\circ} \mathrm{C}$ (Lit. ${ }^{[18 f]}: 170 \sim 175{ }^{\circ} \mathrm{C}$ ); ${ }^{1} \mathrm{H}$ NMR $\left(400 \mathrm{MHz}, \mathrm{CDCl}_{3}\right) \delta$ : 8.93 (br, 1H), 6.65 (dd, $J=7.7,1.2 \mathrm{~Hz}, 1 \mathrm{H}), 6.60 \sim 6.52$ (m, 2H), 6.40 (td, $J=7.5,1.7 \mathrm{~Hz}, 1 \mathrm{H}), 4.45(\mathrm{br}, 2 \mathrm{H}) ;{ }^{13} \mathrm{C}$ NMR $\left(100 \mathrm{MHz}, \mathrm{CDCl}_{3}\right) \delta: 144.0,136.5,119.6,116.5$, 114.5, 114.4; MS (EI) $m / z: 109\left[\mathrm{M}^{+}\right]$.

邻甲基苯酚 $(2 \mathbf{p})^{[19]}$ : 白色固体, m.p. $28 \sim 29{ }^{\circ} \mathrm{C}$ (Lit. $\left.{ }^{[1 \mathrm{f}]}: 30{ }^{\circ} \mathrm{C}\right)$; ${ }^{1} \mathrm{H}$ NMR $\left(400 \mathrm{MHz}, \mathrm{CDCl}_{3}\right) \delta: 7.16 \sim$ $7.09(\mathrm{~m}, 2 \mathrm{H}), 6.90 \sim 6.86(\mathrm{~m}, 1 \mathrm{H}), 6.79(\mathrm{dd}, J=8.0,0.5$ $\mathrm{Hz}, 1 \mathrm{H}), 4.90$ (br, 1H), 2.28 (s, 3H); ${ }^{13} \mathrm{C}$ NMR (100 MHz, $\left.\mathrm{CDCl}_{3}\right) \delta: 153.8,131.2,127.3,123.9,120.9,115.0,15.8$; MS (EI) $m / z: 108\left[\mathrm{M}^{+}\right]$.

邻甲氧基苯酚 $(\mathbf{2 q})^{[19]}$ : 白色固体, m.p. $28 \sim 29{ }^{\circ} \mathrm{C}$ (Lit. $\left.{ }^{[18 g]}: 28 \sim 29{ }^{\circ} \mathrm{C}\right) ;{ }^{1} \mathrm{H}$ NMR $\left(400 \mathrm{MHz}, \mathrm{CDCl}_{3}\right) \delta$ : $6.98 \sim 6.87(\mathrm{~m}, 4 \mathrm{H}), 5.73(\mathrm{~s}, 1 \mathrm{H}), 3.89(\mathrm{~s}, 3 \mathrm{H}) ;{ }^{13} \mathrm{C} \mathrm{NMR}$ $\left(100 \mathrm{MHz}, \mathrm{CDCl}_{3}\right) \delta: 146.7,145.8,121.6,120.2,114.7$, 110.9, 56.0; MS (EI) $m / z: 124\left[\mathrm{M}^{+}\right]$.

邻羟基苯甲酸 $(2 r)^{[18 h]}$ : 白色固体, m.p. 154 $156{ }^{\circ} \mathrm{C}$ (Lit. ${ }^{[18 h]}$ : $155 \sim 157{ }^{\circ} \mathrm{C}$ ); ${ }^{1} \mathrm{H}$ NMR $(400 \mathrm{MHz}$, DMSO- $\left.d_{6}\right) \delta: 13.68(\mathrm{br}, 1 \mathrm{H}), 11.36(\mathrm{br}, 1 \mathrm{H}), 7.79(\mathrm{~d}, J=$ $7.9 \mathrm{~Hz}, 1 \mathrm{H}), 7.50(\mathrm{dd}, J=11.2,4.4 \mathrm{~Hz}, 1 \mathrm{H}) 6.92(\mathrm{dd}, J=$ $19.0,8.1 \mathrm{~Hz}, 2 \mathrm{H}) ;{ }^{13} \mathrm{C}$ NMR $\left(100 \mathrm{MHz}\right.$, DMSO- $\left.d_{6}\right) \delta$ : $171.9,161.1,135.6,130.3,119.2,117.1,112.9$; MS (EI) $\mathrm{m} / z: 138\left[\mathrm{M}^{+}\right]$.

3-羟基吡啶 (2s) ${ }^{[19]}$ : 白色固体, m.p. $125 \sim 126{ }^{\circ} \mathrm{C}$ (Lit. ${ }^{[18 \mathrm{i}]}$ : $125 \sim 127{ }^{\circ} \mathrm{C}$ ); ${ }^{1} \mathrm{H}$ NMR (400 MHz, DMSO- $d_{6}$ ) $\delta$ : $9.90(\mathrm{~s}, 1 \mathrm{H}), 8.14$ (d, $J=2.7 \mathrm{~Hz}, 1 \mathrm{H}), 8.02$ (dd, $J=4.4,1.6$
$\mathrm{Hz}, 1 \mathrm{H}), 7.20 \sim 7.13(\mathrm{~m}, 2 \mathrm{H}) ;{ }^{13} \mathrm{C}$ NMR $(100 \mathrm{MHz}$, DMSO- $\left.d_{6}\right) \delta: 153.7,140.3,138.0,124.2,122.1$; MS (EI) $m / z: 95\left[\mathrm{M}^{+}\right]$.

间甲基苯酚 (2t) ${ }^{[1 \mathrm{lf}]}$ : 无色液体 ${ }^{[12 \mathrm{~d}]} ;{ }^{1} \mathrm{H}$ NMR (400 $\left.\mathrm{MHz}, \mathrm{CDCl}_{3}\right) \delta: 7.16(\mathrm{t}, J=7.6 \mathrm{~Hz}, 1 \mathrm{H}), 6.81 \sim 6.79(\mathrm{~m}$, $1 \mathrm{H}), 6.70 \sim 6.67(\mathrm{~m}, 2 \mathrm{H}), 5.60(\mathrm{~s}, 1 \mathrm{H}), 2.33(\mathrm{~s}, 3 \mathrm{H}) ;{ }^{13} \mathrm{C}$ NMR $\left(100 \mathrm{MHz}, \mathrm{CDCl}_{3}\right) \delta: 155.3,140.0,129.6,121.8$, 116.2, 112.5, 21.4; MS (EI) $m / z: 108\left[\mathrm{M}^{+}\right]$.

间甲氧基苯酚 $(2 \mathbf{u})^{[19]}$ : 无色液体 ${ }^{[18 b]} ;{ }^{1} \mathrm{H}$ NMR (400 $\left.\mathrm{MHz}, \mathrm{CDCl}_{3}\right) \delta: 7.14(\mathrm{t}, J=8.0 \mathrm{~Hz}, 1 \mathrm{H}), 6.54 \sim 6.45(\mathrm{~m}$, $3 \mathrm{H}), 6.02(\mathrm{br}, 1 \mathrm{H}), 3.78(\mathrm{~s}, 3 \mathrm{H}) ;{ }^{13} \mathrm{C}$ NMR $(100 \mathrm{MHz}$, $\left.\mathrm{CDCl}_{3}\right) \delta: 160.9,156.8,130.4,108.2,106.6,101.8,55.4$; MS (EI) $m / z: 124\left[\mathrm{M}^{+}\right]$.

5-甲基-1,3-苯二酚 $(2 \mathbf{v})^{[18 j]}$ : 白色固体, m.p. 105 $107{ }^{\circ} \mathrm{C}$ (Lit. ${ }^{[18 \mathrm{j}]}: 105 \sim 108{ }^{\circ} \mathrm{C}$ ); ${ }^{1} \mathrm{H}$ NMR $(400 \mathrm{MHz}$, DMSO- $\left.d_{6}\right) \delta: 8.04(\mathrm{~s}, 2 \mathrm{H}), 5.03 \sim 5.01(\mathrm{~m}, 3 \mathrm{H}), 1.11(\mathrm{~s}$, $3 \mathrm{H}) ;{ }^{13} \mathrm{C}$ NMR (100 MHz, DMSO- $\left.d_{6}\right) \delta: 158.3,139.3$, 107.2, 99.8, 21.3; MS (EI) $m / z: 124\left[\mathrm{M}^{+}\right]$.

3,4-二甲基苯酚 $(\mathbf{2 w})^{[18 \mathrm{k}]}$ : 白色固体, m.p. $58 \sim 61{ }^{\circ} \mathrm{C}$ (Lit. ${ }^{[18 \mathrm{k}]}: 59 \sim 62{ }^{\circ} \mathrm{C}$ ); ${ }^{1} \mathrm{H}$ NMR $\left(400 \mathrm{MHz}, \mathrm{CDCl}_{3}\right) \delta: 7.00$ $(\mathrm{d}, J=8.1 \mathrm{~Hz}, 1 \mathrm{H}), 6.67(\mathrm{~d}, J=2.6 \mathrm{~Hz}, 1 \mathrm{H}), 6.61(\mathrm{dd}, J=$ $8.1,2.7 \mathrm{~Hz}, 1 \mathrm{H}), 5.36$ (s, 1H), $2.21(\mathrm{~d}, J=8.7 \mathrm{~Hz}, 6 \mathrm{H}) ;{ }^{13} \mathrm{C}$ NMR $\left(100 \mathrm{MHz}, \mathrm{CDCl}_{3}\right) \delta: 153.4,138.1,130.6,128.8$, 116.7, 112.6, 19.9, 18.8; MS (EI) $m / z: 122 \quad\left[\mathrm{M}^{+}\right]$.

5-乙酰-4-羟基-2-甲氧基芐基二异丙基氨基二硫代 甲酸酯(A2) ${ }^{[16]}$ : 白色固体, ${ }^{1} \mathrm{H} \mathrm{NMR}\left(500 \mathrm{MHz}, \mathrm{CDCl}_{3}\right) \delta$ : $12.75(\mathrm{~s}, 1 \mathrm{H}),, 7.86(\mathrm{~s}, 1 \mathrm{H}), 6.39(\mathrm{~s}, 1 \mathrm{H}), 4.51(\mathrm{~s}, 2 \mathrm{H}), 3.87$ (s, 3H), $2.55(\mathrm{~s}, 3 \mathrm{H}), 1.60 \sim 1.33(\mathrm{~m}, 14 \mathrm{H}) .{ }^{13} \mathrm{C}$ NMR $(125$ $\mathrm{MHz}, \mathrm{CDCl}_{3}$ ) $\delta: 202.9,165.0,164.3,133.7,116.5,113.5$, 99.5, 56.1, 26.5, 20.0; MS (EI) $m / z: 355\left[\mathrm{M}^{+}\right]$.

\section{References}

[1] (a) Kwong, H.-K.; Lo, P.-K.; Yiu, S.-M.; Hirao, H.; Lau, K.-C.; Lau, T.-C. Angew. Chem., Int. Ed. 2017, 56, 12260.

(b) Bracegirdle, S.; Anderson, E. A. Chem. Commun. 2010, 46, 3454 .

(c) Ohwada, A.; Nara, S.; Sakamoto, T.; Kikugawa, Y. J. Chem. Soc., Perkin Trans. 1 2001, 3064.

(d) Rayment, E. J.; Summerhill, N.; Anderson, E. A. J. Org. Chem. 2012, 77, 7052 .

(e) Morimoto, Y.; Bunno, S.; Fujieda, N.; Sugimoto, H.; Itoh, S. J. Am. Chem. Soc. 2015, 137, 5867.

(f) Yin, W.; Pan, X.; Leng, W.; Chen, J.; He, H. Green Chem. 2019, $21,4614$.

[2] (a) Nakayama, M.; Fukuoka, Y.; Nozaki, H.; Matsuo, A.; Hayashi, S. Chem. Lett. 1980, 9, 1243.

(b) Rappoport, Z. The Chemistry of Phenols, Wiley, Hoboken, N. J., 2003.

[3] (a) Schmidt, R. J. Appl. Catal. A 2005, 280, 89.

(b) Fyfe, C. A. The Chemistry of the Hydroxyl Group, Vol. 1, Ed.: Patai, S., Wiley-Interscience, New York, 1971, pp. 83 127.

[4] Cornils, B.; Herrmann, W. A. J. Catal. 2003, 216, 23. 
[5] Taddei, M.; Ricci, A. Synthesis 1986, 1986, 633.

[6] (a) Damkaci, F.; Sigindere, C.; Sobiech, T.; Vik, E.; Malone, J. Tetrahedron Lett. 2017, 58, 3559.

(b) Ding, G.; Han, H.; Jiang, T.; Wu, T.; Han, B. Chem. Commun. 2014, 50, 9072 .

(c) Ghiasbeigi, E.; Soleiman-Beigi, M. ChemistrySelect 2019, 4, 3611 .

(d) Mketo, N.; Jordaan, H. J. L.; Jordaan, A.; Swarts, A. J.; Mapolie, S. F. Eur. J. Inorg. Chem. 2016, 3781.

(e) Wang, D.; Kuang, D.; Zhang, F.; Tang, S.; Jiang, W. Eur. J. Org. Chem. 2014, 315.

(f) Xia, S.; Gan, L.; Wang, K.; Li, Z.; Ma, D. J. Am. Chem. Soc. 2016, $138,13493$.

(g) Shendage, S. S. J. Chem. Sci. 2018, 130, 13.

(h) Cyr, P.; Charette, A. B. Synlett 2014, 25, 1409.

(i) Ramu, R.; Wanna, W. H.; Janmanchi, D.; Tsai, Y.-F.; Liu, C.-C.; Mou, C.-Y.; Yu, S.-S. Mol. Catal. 2017, 441, 114.

[7] Fier, P. S.; Maloney, K. M. Org. Lett. 2017, 19, 3033.

[8] Zhao, P.; Zhang, Y.; Li, D.; Cui, H.; Zhang, L. Chin. J. Catal. 2018, 39, 334

[9] (a) Amani, J.; Alam, R.; Badir, S.; Molander, G. A. Org. Lett. 2017, 19, 2426.

(b) Li, Z.; Song, H.; Guo, R.; Zuo, M.; Hou, C.; Sun, S.; He, X.; Sun, Z.; Chu, W. Green Chem. 2019, 21, 3602.

(c) Chen, D.; Liu, J.; Zhang, X.; Jiang, H.; Li, J. Chin. J. Org. Chem. 2019, 39, 3353 (in Chinese).

(陈丹, 刘剑沉, 张馨元, 蒋合众, 李加洪, 有机化学, 2019, 39, 3353.)

(d) Wang, D.; Zhang, L.; Luo, S. Chin. J. Chem. 2018, 36, 311.

[10] (a) Cai, Y.-M.; Xu, Y.-T.; Zhang, X.; Gao, W.-X.; Huang, X.-B.; Zhou, Y.-B.; Liu, M.-C.; Wu, H.-Y. Org. Lett. 2019, 21, 8479.

(b) Hou, T.; Lu, P.; Li, P. Tetrahedron Lett. 2016, 57, 2273.

(c) Xuan, J.; Zhang, Z.-G.; Xiao, W.-J. Angew. Chem., Int. Ed. 2015, 54, 15632.

(d) Xiao, L.; Li, J.; Wang, T. Acta Chim. Sinica 2019, 77, 841 (in Chinese).

(肖丽, 刘嘉恒, 王挺, 化学学报, 2019, 77, 841).

(e) Wang, H.; Wu, P.; Zhao, X.; Zeng, J.; Wang, Q. Acta Chim. Sinica 2019, 77, 231 (in Chinese).

(王浩, 吴品儒, 赵祥, 曾静, 万谦, 化学学报, 2019, 77, 231).

[11] Jiang, M.; Yang, H.; Fu, H. Org. Lett. 2016, 18, 5248.

[12] (a) Zablocka, M.; Hameau, A.; Caminade, A.-M.; Majoral, J.-P. Adv. Synth. Catal. 2010, 352, 2341.

(b) Zhang, M.; Ruzi, R.; Li, N.; Xie, J.; Zhu, C. Org. Chem. Front. 2018, 5, 749 .

(c) Kitanosono, T.; Masuda, K.; Xu, P.; Kobayashi, S. Chem. Rev. 2018, 118,679 .

(d) Song, G.-L.; Zhang, Z.; Da, Y.-X.; Wang, X.-C. Tetrahedron
2015, 71, 8823.

[13] Jiang, M.; Yang, H.; Fu, H. Org. Lett. 2016, 18, 5248.

[14] (a) Ke, F.; Xu, Y.; Zhu, S.; Lin, X.; Lin, C.; Zhou, S.; Su, H. Green Chem. 2019, 21, 4329.

(b) Ke, F.; Liu, C.; Zhang, P.; Xu, J.; Chen, X. Synth. Commun. 2018, 48, 3089.

[15] (a) Tian, Y.-E.; Sun, D.; Han, X.-X.; Yang, J.-M.; Zhang, S.; Feng, N.-N.; Zhu, L.-N.; Xu, Z.-Y.; Che, Z.-P.; Liu, S.-M.; Lin, X.-M.; Jiang, J.; Chen, G.-Q. J. Asian Nat. Prod. Res. 2020, Doi: 10.1080/10286020.2020.1718116.

(b) Peng, Y.; Zheng, X.; Fan, Z.; Zhou, H.; Zhu, X.; Wang, G.; Liu, Z. Phytomedicine 2020, 68, Article 153151.

[16] (a) Cheng, C.-S.; Chen, J.-X; Tang, J.; Geng, Y.-W.; Zheng, L.; Lv, L.-L.; Chen, L.-Y.; Chen, Z. Cancer Manage. Res. 2020, 12, 641.

(b) Huang, W.; Ding, Y.; Miao, Y.; Liu, M.-Z.; Li, Y.; Yang, G.-F. Eur. J. Med. Chem. 2009, 44, 3687.

[17] (a) Cai, Y.-M.; Xu, Y.-T.; Zhang, X. Gao, W.-X.; Huang, X.-B.; Liu, M.-C.; Wu, H.-Y. Org. Lett. 2019, 21, 10169.

(b) Jung, S.-H.; Yeon, J.-W.; Hong, S. Y.; Kang, Y.; Song, K. Nucl. Sci. Eng. 2015, 181, 191.

(c) Banerjee, A.; Lei, Z.; Ngal, M.-Y. Synthesis 2019, 51, 303.

(d) Liu, W.; Li, J.; Huang, C.-Y.; Li, C.-J. Angew. Chem., Int. Ed. 2020, 59, 1786.

(e) Li, L.; Liu, W.; Zeng, H.; Mu, X.; Cosa, G.; Mi, Z.; Li, C.-J. J. Am. Chem. Soc. 2015, 137, 8328.

[18] (a) González-Calderón, D.; González-González, C.-A.; FuentesBenítez, A.; Cuevas-Yáñez, E.; Corona-Becerril, D.; González-Romero, C. Helv. Chim. Acta 2014, 97, 965.

(b) Bora, S. J.; Chetia, B. J. Organomet. Chem. 2017, 851, 52.

(c) Reitti, M.; Gurubrahamam, R., Walther, M.; Lindstedt, E.; Olofsson, B. Org. Lett. 2018, 20, 1785.

(d) Sang, D.; Wang, J.; Zheng, Y.; He, J.; Yuan, C.; An, Q.; Tian, J. Synthesis 2017, 49, 2721.

(e) Ghorbani-Choghamarani, A.; Goudarziafshar, H.; Nikoorazm, M.; Naseri Z. Chin. Chem. Lett. 2011, 22, 1431.

(f) Rahimi, J.; Taheri-Ledari, R.; Niksefat, M.; Maleki A. Catal. Commun. 2020, 134, 105850

(g) Xu, J.; Wang, X.; Shao, C.; Su, D.; Cheng, G..; Hu, Y. Org. Lett. 2010, 12, 1964.

(h) Sodhi, R. K.; Paul, S.; Clark, J. H. Green Chem. 2012, 14, 1649. (i) Chatterjee, N.; Chowdhury, H.; Sneh, K.; Goswami A. Tetrahedron Lett. 2015, 56, 172.

(j) Richter, H.; Beckendorf, S.; Mancheño, O. G. Adv. Synth. Catal. 2011, 353, 295.

(k) Sridhar, A.; Rangasamy, R.; Selvaraj, M. New J. Chem. 2019 43, 17974.

[19] Jing, L.; Wei, J.; Zhou, L.; Li, Z.; Zhou, X. Chem. Commun. 2010 , 46, 4767. 